\title{
Synthesis, structure and low temperature study of electric transport and magnetic properties of $\mathrm{GdSr}_{2} \mathrm{MnCrO}_{7}$
}

\author{
DEVINDER SINGH*, MEENA SHARMA, SUSHMA SHARMA and RAJINDER SINGH \\ Department of Chemistry, University of Jammu, Jammu 180 006, India
}

MS received 11 October 2010; revised 9 December 2010

\begin{abstract}
The layered perovskite oxide, $\mathrm{GdSr}_{2} \mathrm{MnCrO}_{7}$, has been prepared by the standard ceramic method. The powder X-ray diffraction studies suggest that the phase crystallizes with tetragonal unit cell in the space group $I 4 / \mathrm{mmm}$. The electrical transport properties show that the phase is an electrical insulator and the electrical conduction in the phase occurs by a 3D variable range hopping mechanism. The magnetic properties suggest that the ferromagnetic interactions are dominant in the high temperature region.
\end{abstract}

Keywords. Chemical synthesis; X-ray diffraction; electrical properties; magnetic properties.

\section{Introduction}

Layered perovskite oxides are a promising group of mixedconducting materials with potential applications for oxygenseparation membranes, gas sensor devices and electrodes of intermediate-temperature solid oxide fuel cells (Moseley and Williams 1989; Meixner and Lampe 1996; Skinner and Kilner 2000; Ormerod 2003). These types of oxides with composition, $\mathrm{La}_{2-x} \mathrm{M}_{1+x} \mathrm{Mn}_{2} \mathrm{O}_{7}(\mathrm{M}=\mathrm{Ca}, \mathrm{Sr}, \mathrm{Ba})$, have attracted particular attention of researchers owing to their remarkable properties, including electronic and impurity phase separation, giant magnetoresistance, and some others (Kimura and Tokura 2000; Dagotto et al 2001). In general, the layered perovskite can be represented as Ruddlesden-Popper (RP) series of general formula, $\mathrm{A}_{n+1} \mathrm{~B}_{n} \mathrm{O}_{3 n+1}$ or $\mathrm{AO} \cdot\left(\mathrm{ABO}_{3}\right)_{n}$, where $n$ is the number of perovskite blocks composed of twodimensional layers of $\mathrm{BO}_{6}$ corner-sharing octahedra separated by rock salt AO layers (Ruddlesden and Popper 1957, 1958), A is a rare earth element and B is a transition element. A number of $n=2$ members of RP rare earth lanthanum manganites and their substituted analogues have been reported in the literature (Jung 2000, 2003; Arbuzova et al 2003; Sudhakar and Rajeev 2004; Zhang et al 2004; Yankin et al 2006; Fedorova et al 2008). Although there are many systematic studies on the hole-doped manganites in which concentration of the alkaline earth metal ion is varied, thereby affecting the $\mathrm{Mn}^{3+} / \mathrm{Mn}^{4+}$ ratio, which has a direct bearing on the double exchange mechanism responsible for CMR, there are far fewer studies on other rare earth manganites and the doping at the Mn site in these systems. Notable among these are doping with various transition elements, such as Fe, Cr, Ni, Co and Ru at the Mn site. Only Ru-doped systems show enhanced FM and metallicity while rest of the

*Author for correspondence (drdssambyal@rediffmail.com) dopants destroyed the FM (Gundakaram et al 1999; Zhang et al 1999, 2000; Raveau et al 2000; Maignan et al 2001; Martin et al 2001; Sahu et al 2002; Sudhakar et al 2003).

In the present work, a new RP-type phase, $\mathrm{GdSr}_{2} \mathrm{MnCrO}_{7}$ has been synthesized and its crystal structure has been determined by powder X-ray diffractometry. The electrical resistivity and magnetic susceptibility of the phase have been studied as functions of temperature.

\section{Experimental}

$\mathrm{GdSr}_{2} \mathrm{MnCrO}_{7}$ was prepared by the standard ceramic method by heating stoichiometric amounts of $\mathrm{MnO}_{2}$ (Aldrich 99.9\%), $\mathrm{Cr}_{2} \mathrm{O}_{3}$ (Aldrich 99.9\%), $\mathrm{Gd}_{2} \mathrm{O}_{3}$ (Aldrich 99.9\%), except $\mathrm{SrCO}_{3}$ (Aldrich 99.9\%), 5\% of which was added in excess to compensate for its loss at high temperature. Prior to use, $\mathrm{Gd}_{2} \mathrm{O}_{3}$ was heated at $1000^{\circ} \mathrm{C}$ to remove moisture. Before heating, the mixtures were homogenized by grinding and pressed into pellets. The pellets were heat-treated at $1553( \pm 10) \mathrm{K}$ in static air for about $72 \mathrm{~h}$ with a number of intermittent grindings and pelletizings. Finally, the sample was cooled down slowly to room temperature in the electric furnace. The black coloured product was finally pulverized. The amount of cations in the phase was determined by quantitative analysis within an accuracy of $\sim 2 \%$. The oxygen content in the sample was determined by iodometric method. The precise chemical composition was determined to be $\mathrm{GdSr}_{2} \mathrm{MnCrO}_{6.93}$.

The room temperature X-ray diffraction data of the phase was recorded on Bruker AXS diffractometer type D 76181 (Karlsruhe, Germany) using $\mathrm{CuK}_{\alpha}$ radiations. The data were collected at a scanning speed of $1 \% \mathrm{~min}$ and in the $2 \theta$ range of $10-80^{\circ}$. The experimental XRD data is given in table 1 , while the pattern is plotted in figure 1 . 


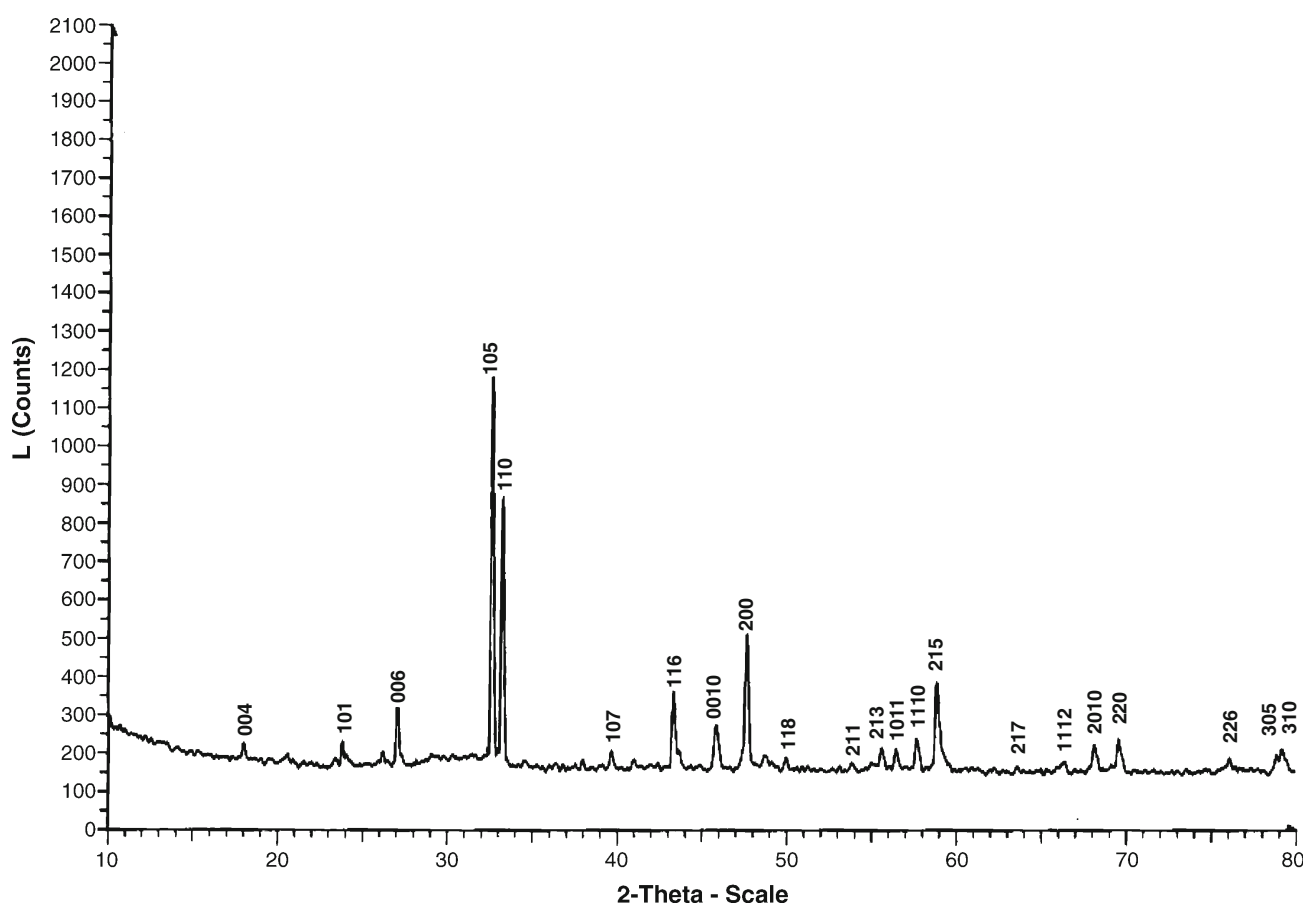

Figure 1. X-ray diffraction pattern of $\mathrm{GdSr}_{2} \mathrm{MnCrO}_{7}$.

The electrical resistivity measurements of the pellet of the product, sintered at $1400 \mathrm{~K}$ for about $12 \mathrm{~h}$ before use, were carried out with four probe method in the temperature range $150-300 \mathrm{~K}$. For the electrodes, thin copper wires were attached to the surface of the pellet with silver paste. The magnetic susceptibility of the polycrystalline sample was measured by means of the Faraday technique in the temperature range $100-300 \mathrm{~K}$ using $\mathrm{Hg}\left[\mathrm{Co}(\mathrm{SCN})_{4}\right]$ as calibrant in an external field of 3,700 gauss. All the magnetic susceptibility values were corrected for diamagnetism of the constituent atoms.

\section{Results and discussion}

All the peaks of X-ray diffraction pattern of polycrystalline sample, $\mathrm{GdSr}_{2} \mathrm{MnCrO}_{7}$, were successfully indexed on the tetragonal $\mathrm{Sr}_{3} \mathrm{Ti}_{2} \mathrm{O}_{7}$-type structure of space group $14 / \mathrm{mmm}$. The unit cell parameters, refined from the program 'Unit Cell', are listed in table 1. The atomic positions of $\mathrm{Gd}, \mathrm{Sr}$, $\mathrm{Mn}, \mathrm{Cr}$ and $\mathrm{O}$ were generated for $\mathrm{GdSr}_{2} \mathrm{MnCrO}_{7}$ from the known positional parameters of $\mathrm{Sr}_{3} \mathrm{~V}_{2} \mathrm{O}_{7}$ (Suzuki et al 1991) and are given in table 2 . The theoretical diffraction intensities (table 1) of the phase were generated with a program 'Mercury' based on these atomic positions, cell parameters and space group $14 / \mathrm{mmm}$. The agreement between the theoretical and experimental intensities for the phase is, in general, satisfactory considering that the atomic positions are not refined and that, any preferred orientation effects are neglected. The quantitative analysis, oxygen stoichiometry, the agreement between $d_{\mathrm{obs}}$ and $d_{\mathrm{cal}}$ and that between $I_{\mathrm{obs}}$ and $I_{\mathrm{cal}}$ values
Table 1. Powder X-ray diffraction data of $\mathrm{GdSr}_{2} \mathrm{MnCrO}_{7}$ (Space group: I4/mmm, $a=3 \cdot 8177(4) \AA$ and $c=19 \cdot 7977(5) \AA$ ).

\begin{tabular}{rrrrrrr}
\hline$h$ & $k$ & $l$ & $d_{\text {obs }}(\AA)$ & $d_{\text {cal }}(\AA)$ & $I_{\text {obs }}$ & $I_{\text {cal }}$ \\
\hline 0 & 0 & 4 & 4.941 & 4.949 & 5 & 6 \\
1 & 0 & 1 & 3.742 & $3 \cdot 748$ & 20 & 34 \\
0 & 0 & 6 & 3.298 & $3 \cdot 299$ & 29 & 31 \\
1 & 0 & 5 & $2 \cdot 746$ & $2 \cdot 748$ & 100 & 100 \\
1 & 1 & 0 & $2 \cdot 697$ & $2 \cdot 699$ & 74 & 78 \\
1 & 0 & 7 & $2 \cdot 272$ & $2 \cdot 272$ & 17 & 48 \\
1 & 1 & 6 & $2 \cdot 088$ & $2 \cdot 089$ & 30 & 34 \\
0 & 0 & 10 & 1.979 & 1.979 & 23 & 52 \\
2 & 0 & 0 & 1.907 & 1.908 & 43 & 44 \\
1 & 1 & 8 & 1.824 & 1.824 & 16 & 17 \\
2 & 1 & 1 & 1.701 & 1.701 & 14 & 18 \\
2 & 1 & 3 & 1.652 & 1.652 & 18 & 15 \\
1 & 0 & 11 & 1.628 & 1.627 & 17 & 15 \\
1 & 1 & 10 & 1.597 & 1.596 & 20 & 31 \\
2 & 1 & 5 & 1.568 & 1.567 & 32 & 46 \\
2 & 1 & 7 & 1.461 & 1.461 & 14 & 15 \\
1 & 1 & 12 & 1.407 & 1.407 & 15 & 14 \\
2 & 0 & 10 & 1.374 & 1.374 & 18 & 17 \\
2 & 2 & 0 & 1.350 & 1.349 & 20 & 21 \\
2 & 2 & 6 & 1.249 & 1.249 & 3 & 4 \\
3 & 0 & 5 & 1.214 & 1.212 & 6 & 4 \\
3 & 1 & 0 & 1.207 & 1.207 & 9 & 13 \\
\hline & & & & & &
\end{tabular}

suggests that the phase with composition, $\mathrm{GdSr}_{2} \mathrm{MnCrO}_{6.93}$, having $\mathrm{Sr}_{3} \mathrm{Ti}_{2} \mathrm{O}_{7}$ type structure has been formed.

Selected interatomic bond lengths and bond angles have also been calculated from the structural parameters, which 
Table 2. Structural parameters of $\mathrm{GdSr}_{2} \mathrm{MnCrO}_{7}$.

\begin{tabular}{lcccc}
\hline Atom & Site & $x$ & $y$ & $z$ \\
\hline \multicolumn{4}{l}{ Positional coordinates of Gd, Sr, Mn, Cr and O (Space group, $14 / \mathrm{mmm})$} \\
$\mathrm{Gd} / \mathrm{Sr}(1)$ & $2 \mathrm{~b}$ & $0 \cdot 0$ & $0 \cdot 0$ & 0.5 \\
$\mathrm{Gd} / \mathrm{Sr}(2)$ & $4 \mathrm{e}$ & $0 \cdot 0$ & $0 \cdot 0$ & $0.3148(3)$ \\
$\mathrm{Mn} / \mathrm{Cr}$ & $4 \mathrm{e}$ & $0 \cdot 0$ & $0 \cdot 0$ & $0.0971(7)$ \\
$\mathrm{O}(1)$ & $2 \mathrm{a}$ & $0 \cdot 0$ & $0 \cdot 0$ & 0.0 \\
$\mathrm{O}(2)$ & $4 \mathrm{e}$ & $0 \cdot 0$ & $0 \cdot 0$ & $0.190(2)$ \\
$\mathrm{O}(3)$ & $8 \mathrm{~g}$ & $0 \cdot 0$ & 0.5 & $0.096(2)$
\end{tabular}

Selected bond lengths $(\AA)$

$\begin{array}{lccc}\mathrm{Gd} / \mathrm{Sr}(2)-\mathrm{O}(2) & 2.491(40) & \mathrm{Gd} / \mathrm{Sr}(2)-\mathrm{O}(2) & 2.711(1) \\ \mathrm{Gd} / \mathrm{Sr}(2)-\mathrm{O}(3) & 2.615(27) & \mathrm{Gd} / \mathrm{Sr}(2)-\mathrm{O}(3) & 2.710(28) \\ \mathrm{Gd} / \mathrm{Sr}(2)-\mathrm{O}(1) & 2.709(0) & \mathrm{Mn} / \mathrm{Cr}-\mathrm{O}(2) & 1.854(42) \\ \mathrm{Mn} / \mathrm{Cr}-\mathrm{O}(1) & 1.938(14) & \mathrm{Mn} / \mathrm{Cr}-\mathrm{O}(3) & 1.916(0)\end{array}$

Bond angles $\left({ }^{\circ}\right)$

$\begin{array}{lccc}\mathrm{O}(2)-\mathrm{Mn} / \mathrm{Cr}-\mathrm{O}(3) & 90.66(105) & \mathrm{O}(1)-\mathrm{Mn} / \mathrm{Cr}-\mathrm{O}(3) & 89.34(77) \\ \mathrm{O}(2)-\mathrm{Mn} / \mathrm{Cr}-\mathrm{O}(1) & 179.99(86) & \mathrm{Gd} / \mathrm{Sr}(1)-\mathrm{Gd} / \mathrm{Sr}(2)-\mathrm{O}(2) & 180(0) \\ \mathrm{Mn} / \mathrm{Cr}-\mathrm{O}(1)-\mathrm{Mn} / \mathrm{Cr} & 180(34) & \mathrm{Mn} / \mathrm{Cr}-\mathrm{O}(3)-\mathrm{Mn} / \mathrm{Cr} & 178.69(42)\end{array}$
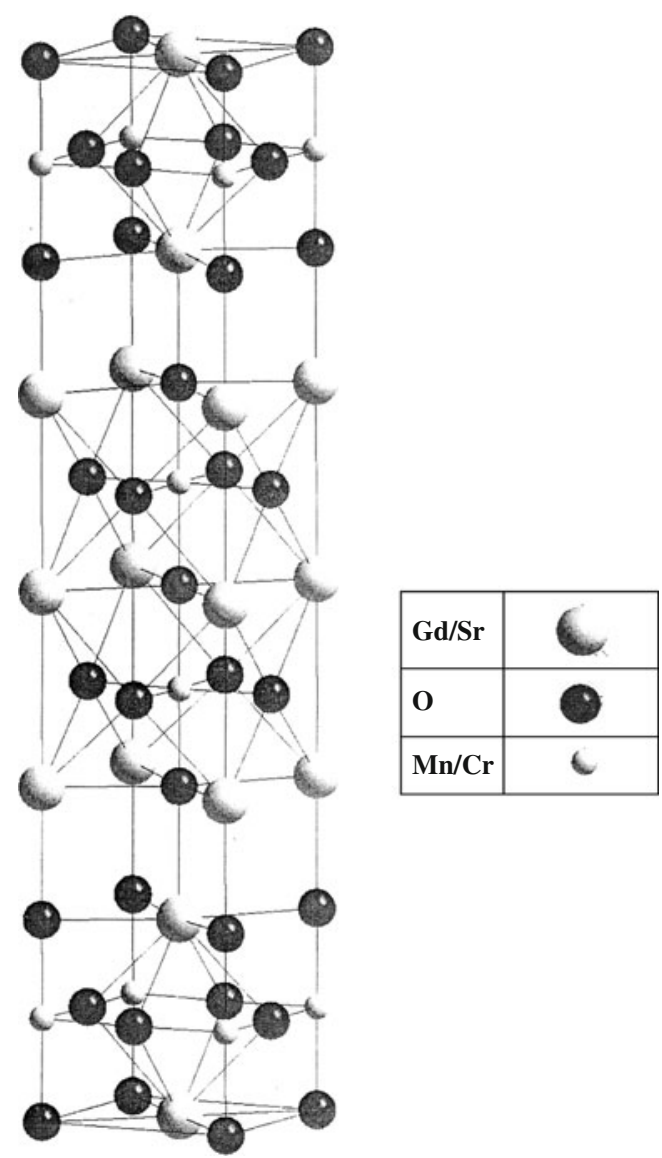

Figure 2. Unit cell structure of $\mathrm{GdSr}_{2} \mathrm{MnCrO}_{7}$.

are tabulated in table 2 . The average coordination geometry about the transition metal $(\mathrm{Mn} / \mathrm{Cr})$ site is somewhat irregular, which is often observed in the RP-type phases (Attfield

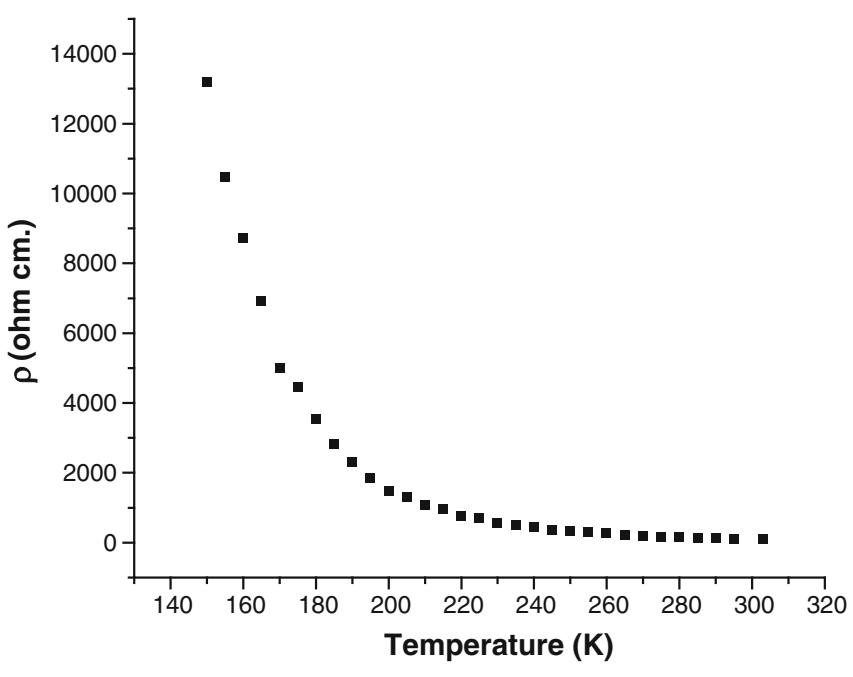

Figure 3. Plot of $\rho$ vs temperature (K) of $\mathrm{GdSr}_{2} \mathrm{MnCrO}_{7}$.

et al 1992; Battle et al 1992). One of the reasons for this asymmetry in the present case seems to be the presence of the mixed valence state of the manganese ion $\left(\mathrm{Mn}^{3+} / \mathrm{Mn}^{4+}\right)$ in the lattice. The mixed valence state of $\mathrm{Mn}$ could be due to the oxygen deficiency in the phase. The $\mathrm{Mn} / \mathrm{Cr}-\mathrm{O}(1)$ bond is stretched and longest of all are the $\mathrm{Mn} / \mathrm{Cr}-\mathrm{O}$ bonds in $\mathrm{GdSr}_{2} \mathrm{MnCrO}_{7}$. This implies that the structure has the freedom to adapt along the $c$-axis. The unit cell structure of the phase as drawn with the program 'Diamond' is given in figure 2 .

The temperature dependence of electrical resistivity is given in figure 3, where $\rho$ is plotted against temperature $(T)$. The plot shows that the temperature coefficient of resistivity is negative suggesting that the material is an insulator in 
the temperature range $150-300 \mathrm{~K}$. The linearity of $\log \rho$ versus $T^{-1 / 4}$ plot (figure 4 ) in the temperature of investigation shows that the electronic conduction occurs by a $3 \mathrm{D}$ variable range hopping (VRH) mechanism expressed by

$$
\rho=\rho_{0} \exp \left(B T^{-1 / 4}\right)
$$

The insulator behaviour is attributed to the superexchange coupling of electrons. Such type of manganites show insulator-metal (I-M) transition at low temperatures but our study is, however, limited to resistivity measurements in the temperature range 150-300 K.

The temperature dependence of the inverse molar magnetic susceptibility for $\mathrm{GdSr}_{2} \mathrm{MnCrO}_{7}$ is shown in figure 5 .

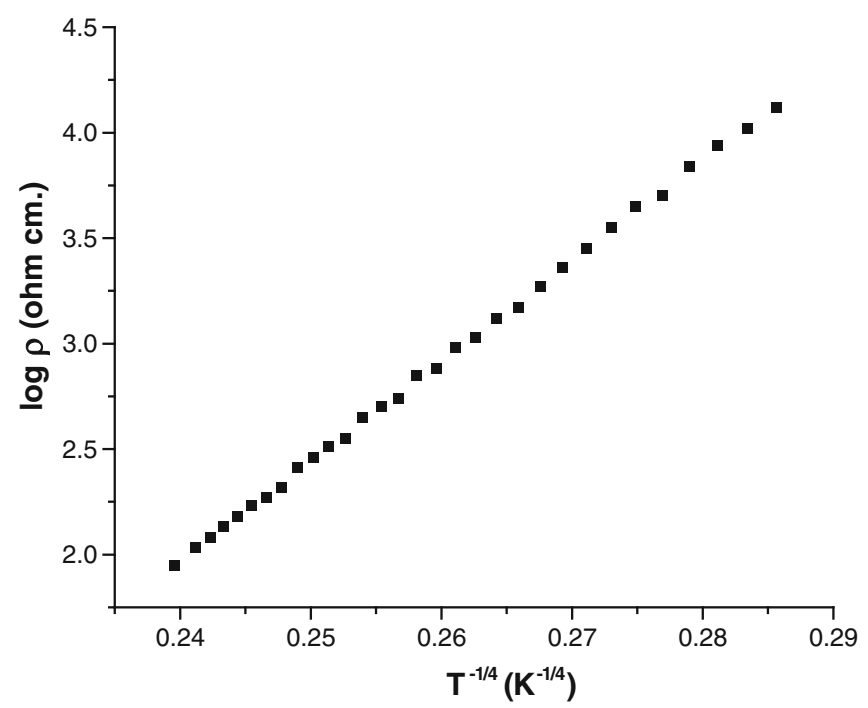

Figure 4. Plot of $\log \rho$ vs $T^{-1 / 4}$ of $\mathrm{GdSr}_{2} \mathrm{MnCrO}_{7}$.

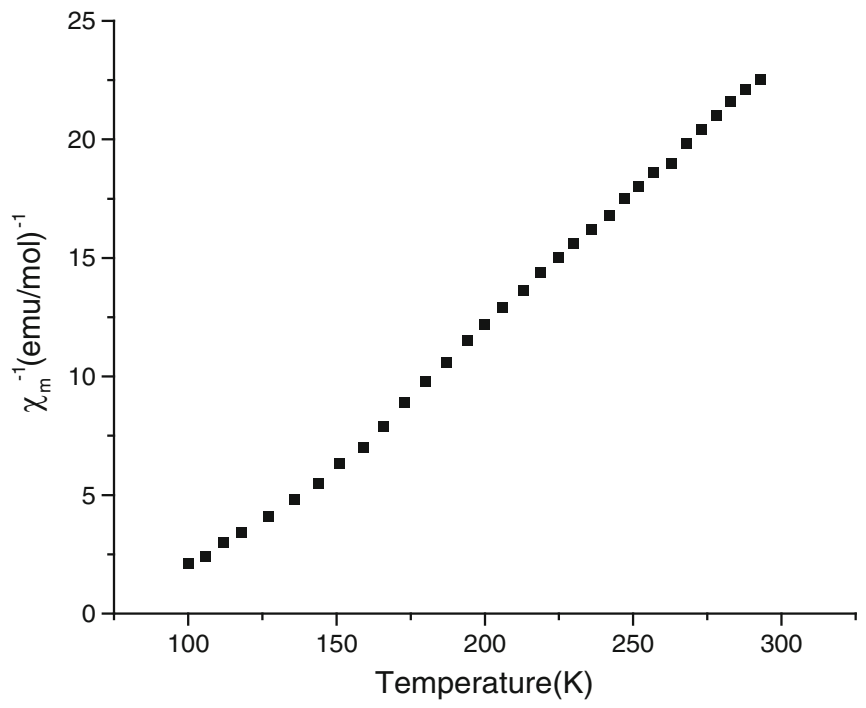

Figure 5. Plot of inverse molar susceptibility $\left(\chi_{\mathrm{M}}^{-1}\right)$ vs temperature $(K)$ of $\mathrm{GdSr}_{2} \mathrm{MnCrO}_{7}$.
The effective magnetic moment $\left(\mu_{\text {eff }}\right)$ has been calculated from the high temperature linear region of the $\chi_{\mathrm{M}}^{-1}$ versus $T$ plot and leads to a value of $9.81 \mathrm{~B}$. M. The contribution of the manganese ion $\left(\mu_{\mathrm{Mn}}\right)$ to the magnetic moment has been calculated from the effective moment $\left(\mu_{\text {eff }}\right)$ and the theoretical magnetic moments of $\mathrm{Gd}^{3+}$ and $\mathrm{Cr}^{3+}$ ions from the relation (Subramanian et al 1988)

$$
\mu_{\mathrm{eff}}^{2}=n_{1} \mu_{\mathrm{Gd}^{3+}}^{2}+n_{2} \mu_{\mathrm{Mn}}^{2}+n_{3} \mu_{\mathrm{Cr}^{3+}}^{2},
$$

where $n_{1}, n_{2}$ and $n_{3}$ are the number of $\mathrm{Gd}, \mathrm{Mn}$ and $\mathrm{Cr}$ ions, respectively. $\mu_{\mathrm{Gd}^{3}}$ is the theoretical magnetic moment of the $\mathrm{Gd}^{3+}$ ion (7.94 B.M.) and $\mu_{\mathrm{Cr}^{3+}}$ is that of $\mathrm{Cr}^{3+}$ ion, assuming it to be in the high spin state $\left(t_{2 g}^{3} e_{g}^{0}\right)$. For $\mu_{\mathrm{Mn}}$, we obtain a value of $4.26 \mathrm{~B}$. M. This value is larger than the theoretical magnetic moment of high spin $\mathrm{Mn}^{4+}$ ion (3.87 B. M.) which suggests that manganese ion is partly present in $3+$ oxidation state. The stoichiometry of the phase $\left(\mathrm{GdSr}_{2} \mathrm{MnCrO}_{6.93}\right)$ also suggests the presence of manganese ion in mixed valence state $4+$ and $3+(93: 7$ by \%), assuming $\mathrm{La}$ in $3+, \mathrm{Sr}$ in $2+$ and $\mathrm{Cr}$ in $3+$ oxidation states. It has been observed from the $\chi_{\mathrm{M}}^{-1}$ vs $T$ plot (figure 5) that the Weiss constant $(\theta)$ obtained from the high temperature linear region is $56 \mathrm{~K}$. The positive $(\theta)$ value suggests that ferromagnetic interactions are dominant in the magnetic structure of $\mathrm{GdSr}_{2} \mathrm{MnCrO}_{7}$. The ferromagnetic curie temperature $\left(T_{\mathrm{c}}\right)$ obtained from the molar magnetic susceptibility $\left(\chi_{M}\right)$ vs temperature plot was found to be $140 \mathrm{~K}$.

The ferromagnetic interactions could be due to double exchange (DE) interaction between $\mathrm{Mn}^{3+}$ and $\mathrm{Mn}^{4+}$ ions. Since the $\mathrm{Cr}^{3+}$ ion has the same electronic configuration $\left(t_{2 g}^{3} e_{g}^{0}\right)$ as the $\mathrm{Mn}^{4+}$ ion, there may exist a ferromagnetic DE interaction between $\mathrm{Cr}^{3+}$ and $\mathrm{Mn}^{3+}$ ions just as between $\mathrm{Mn}^{4+}$ and $\mathrm{Mn}^{3+}$ ions. The proposal has been proved by some experimental results (Maignan et al 1998).

\section{Conclusions}

A new RP-type phase of composition, $\mathrm{GdSr}_{2} \mathrm{MnCrO}_{7}$, has been synthesized by standard ceramic method. Its structure has been determined by X-ray diffractometry. The results show that the phase crystallizes in the $14 / \mathrm{mmm}$ space group with tetragonal unit cell. The electrical resistivity studies show that the material is an electrical insulator and conduction occurs by a variable range hopping mechanism. The magnetic studies suggest that the ferromagnetic interactions are dominant and manganese ion in the phase is present in mixed valence state $\left(\mathrm{Mn}^{3+} / \mathrm{Mn}^{4+}\right)$.

\section{Acknowledgements}

Thanks are due to Prof. A Ramanan, Department of Chemistry, Indian Institute of Technology, New Delhi, for providing computer programmes for structure determination. 
We are also thankful to Prof. Ramesh Chandra, Institute Instrumentation Centre, Indian Institute of Technology, Roorkee, for collecting XRD data.

\section{References}

Arbuzova T I, Naumov S V and Arbuzov V L 2003 Phys. Solid State 451513

Attfield M P, Battle P D, Bollen S K, Kin S H, Powell A V and Worlman M 1992 J. Solid State Chem. 96344

Battle P D, Bollen S K and Powell A V 1992 J. Solid State Chem. 99267

Dagotto E, Hotta T and Moreo A 2001 Phys. Rep. 3441

Fedorova M O, Yankin A M, Zvereva I A, Titova S G and Balakirev V F 2008 Glass Phys. Chem. 34201

Gundakaram R, Lin J G, Lee F Y, Tai M F, Shen C H, Liu R S and Huang C Y 1999 J. Phys.: Condens. Matter 115187

Jung W -H 2000 J. Mater. Sci. Lett. 191307

Jung W -H 2003 J. Mater. Sci. Lett. 22527

Kimura T and Tokura Y 2000 Ann. Rev. Mater. Sci. 30451

Maignan A, Martin C, Damay F, Hervien M and Raveau B 1998 J. Magn. Magn. Mater. 188185

Maignan A, Martin C, Hervien M and Raveau B 2001 Solid State Commun. 117377
Martin C, Maignan A, Hervien M, Antret C, Raveau B and Khomski D I 2001 Phys. Rev. B63 174404

Meixner H and Lampe U 1996 Sensor Actuator B33 198

Moseley P T and Williams D E 1989 Polyhedron 91615

Ormerod R M 2003 Chem. Soc. Rev. 3217

Raveau R, Maignan A, Martin C, Mahendiran R and Hervien M 2000 J. Solid State Chem. 151330

Ruddlesden S N and Popper P 1957 Acta Crystallogr. 10538

Ruddlesden S N and Popper P 1958 Acta Crystallogr. 1154

Sahu R K, Mohammad Q, Rao M L, Manoharan S S and Nigam A K 2002 Appl. Phys. Lett. 8088

Skinner S J and Kilner J A 2000 Solid State Ionics 135709

Subramanian M A, Torardi C C, Johnson D C, Pannetier J and Sleight A W 1988 J. Solid State Chem. 7224

Sudhakar N and Rajeev K P 2004 J. Electron. Mater. 331259

Sudhakar N, Rajeev K P and Nigam A K 2003 J. Appl. Phys. 93 8331

Suzuki N, Noritake T, Yamamoto N and Hioki T 1991 Mater. Res. Bull. 261

Yankin A M, Fedorova O M, Zvereva I A, Titova S G and Balakirev V F 2006 Glass Phys. Chem. 32574

Zhang J, Wang F, Zhang P and Yan Q 1999 J. Appl. Phys. 861604

Zhang J, Yan J Q, Wang F, Yuan P and Zhang P 2000 J. Phys.: Condens. Matter 121981

Zhang R L, Zhao B C, Song W H, Ma Y Q, Yang J, Sheng Z G and Dai J M 2004 J. Appl. Phys. 964965 